\title{
Neuropsychology of the Social Brain Hypothesis of Schizo- phrenia: Symptoms, Personality and MRI Correlates
}

Paul G. Nestor ${ }^{1,2^{*}}$, Margaret Niznikiewicz ${ }^{2,3}$, Victoria Choate Hasler ${ }^{1}$, Dominick T. Newell ${ }^{1,3}$, Martha E. Shenton ${ }^{2,3}$ and Robert W. McCarley ${ }^{2}$

${ }^{1}$ Department of Psychology, University of Massachusetts, Boston, MA, USA

${ }^{2}$ Clinical Neuroscience Division, Laboratory of Neuroscience, Department of Psychiatry, Veterans Affairs (VA) Boston Healthcare System, Harvard Medical School Brockton, MA, USA

${ }^{3}$ Psychiatry Neuroimaging Laboratory, Department of Psychiatry, Brigham and Women's Hospital, Harvard Medical School, Boston, MA, USA

Correspondence to:

Paul G. Nestor

Department of Psychology

University of Massachusetts, Boston

MA 02125-3393, USA

Tel: 617-287-6387

E-mail: paul.nestor@umb.edu

Received: March 15, 2017

Accepted: May 11, 2017

Published: May 17, 2017

Citation: Nestor PG, Niznikiewicz M, Hasler VC, Newell DT, Shenton ME, et al. 2017. Neuropsychology of the Social Brain Hypothesis of Schizophrenia: Symptoms, Personality and MRI Correlates. J Neuroimaging Psychiatry Neurol 2(1): 3-10.

Copyright: ( 2017 Nestor et al. This is an Open Access article distributed under the terms of the Creative Commons Attribution 4.0 International License (CC-BY) (http://creativecommons. org/licenses/by/4.0/) which permits commercial use, including reproduction, adaptation, and distribution of the article provided the original author and source are credited.

Published by United Scientific Group

\begin{abstract}
We examined the social brain hypothesis in schizophrenia using a comprehensive battery of measures of intelligence, personality, and symptoms in a large sample of medicated, long-term, chronic patients. A smaller subset also had available MRI studies of superior temporal gyrus (STG) and fusiform gyrus (FG), regions that have been linked to social cognition in both healthy and clinical samples. Results indicated that patients divided into high- and low-IQ groups differed significantly on proxy measures of social cognition, specifically symptom ratings and personality test scores. That is, high-and low-IQ groups had similar levels of negative and positive symptoms, but showed opposite patterns for personality traits of neuroticism and openness, with the former reduced and the latter elevated in the high-IQ group. Likewise, for the entire sample, reduced neuroticism and elevated openness corresponded with higher ratings on the positive symptom of formal thought disturbance. MRI data also revealed evidence of distinct symptom-personality relationships marked by decreased gray matter volumes in the: (a) STG with increased positive symptoms of hallucinations and lower agreeableness and (b) FG with increased negative symptoms of social anhedonia and lower openness. Together, these behavioral and MRI data may help to define the nature of social cognitive disturbance in schizophrenia.
\end{abstract}

\section{Keywords}

Social brain hypothesis, Schizophrenia, Cognition, Personality, Symptoms

\section{Introduction}

Schizophrenia is well known for the heterogeneity of its phenotype expression. Indeed, cognitive deficits, as central to the disease as to be described as the "primary expression of the schizophrenic brain" [1], do not occur in isolation, but rather are accompanied by marked changes in sociality that often lead to significant difficulties in daily interactions with others. Kraepelin [2, 3], and Kretschmer [4] each emphasized these changes as important in the course of the illness. But not until some 40 years later did P. E. Meehl [5] provide, perhaps, the seminal contribution towards understanding the critical role of sociality in the development of schizophrenia. Meehl introduced as a latent genetic liability for schizophrenia, the construct of schizotypy, a personality syndrome defined by a particular set of cognitive and social traits that were hypothesized to represent the behavioral expression of disease vulnerability originating from an inherited central nervous system integrative defect identified as "schizotaxia" [6, 7]. Over 
the next several decades and through to the present, Meehl's formulation would remain a dominant influence in the everburgeoning studies of risk factors in schizophrenia.

More recently, disease-related changes in sociality and cognition have been examined through the lens of evolutionary neuroscience [8-13]. From this perspective, cognitive and social processes are tightly linked, co-evolving to favor a host of vital specialized functional adaptations that advanced fitness by promoting effective human transactions [14]. These processes entail a complex set of representations of internal bodily states, knowledge of self, perceptions of others, and interpersonal motivations, all of which endow the primate brain with its remarkable facility for social cognition [15-17]. These social cognitive abilities are, in turn, supported by a widely-distributed network of diverse brain regions, including the temporoparietal junction, the temporal sulcus and the temporal poles as well as medial prefrontal cortex, especially orbital frontal sections $[12,18]$. These abilities and their underlying neural structures are hypothesized to form the social brain. This in turn is often described as the social brain hypothesis of evolutionary neuroscience, which posits that primates' exceptionally large brains and the massive expansion of their neocortex volumes are the products of the cognitive demands of living in complexly bonded social groups [8]. Notable empirical support for this hypothesis includes neuroimaging studies that have correlated increased gray matter volume with larger social networks in macaques [19] as well as experimental behavioral evidence showing that tasks of social (e.g., observational learning) but not physical (e.g., spatial memory) cognition distinguished 2.5-year old human children from their nearest primate relatives, chimpanzees and orangutans [20-22].

Accordingly, we combined multiple measures of cognition, personality, and symptoms to examine dynamics of social cognition in schizophrenia. To address the wellknown problem of heterogeneity in illness expression, we used neuropsychological tests of intelligence that offer reliable and valid comprehensive assessment of cognitive abilities that are frequently disrupted by the disease [23] and that may also represent key risk factors for schizophrenia [24]. These measures provided a rather simple and straightforward objective tool (median split) to divide patients into highand low-IQ groups. This in turn allowed us to examine these subgroups in terms of individual differences in personality using the influential Costa and $\mathrm{McCrae}$ [25] five-factor model of personality (neuroticism, extraversion, openness, agreeableness, and conscientiousness). The underlying social cognitive processes of these normal, presumably universal personality traits have become an increasingly rich area of research as have studies of their neural correlates [17, 26]. However little is known as to how personality may intersect with intelligence and symptomatology in the social cognitive disturbance of schizophrenia. This is despite evidence suggesting that the personality trait of neuroticism, characterized by emotional instability and anxiety proneness, as a moderating factor, potentiates clinical outcomes among both schizotypic $[7,27]$ and non-clinical [28] samples.

Symptoms represent yet another component of social cognitive disturbance in schizophrenia. Hoffman [29], for example, proposed that positive and negative schizophrenic symptoms can be traced to deafferentation of key structures and functions of the social brain. That is, he theorized that a loss of social inputs, as reflected in severe negative symptoms of emotional withdrawal and isolation in high-risk individuals, disrupts neural and perceptual processes that, in turn, lead to the emergence of positive symptoms of delusions and hallucinations. In Hoffman's conceptualization, just as sensory deafferentation following sensory loss is known to lead to massive cortical reorganization, so too might extreme social withdrawal during critical developmental periods induce deafferentation-like processes in neural circuitry and perceptual process of the social brain of persons at-risk for schizophrenia. These disruptions in the social brain, Hoffman hypothesized, would reflect "spurious social meanings in the form of complex, emotionally compelling hallucinations and delusions representing other persons or agents." (p. 1066).

Other empirical studies have examined the relationship of symptoms and social role function in at-risk samples. For example, Valmaggia et al. [30] provided evidence derived from cluster analysis suggesting that the severest risk type for schizophrenia may be characterized by negative symptoms of alogia, avolition/apathy, anhedonia, and social isolation, which were accompanied by impaired role functioning. Other findings have linked heightened clinical risk for schizophrenia to hypersensitivity to interpersonal interactions as well as to positive symptoms of psychosis [31]. These data from risk studies suggest potential important interactions of symptoms and social functioning in the development of the illness. But whether evidence of these relationships also holds in the full expression of the illness is unclear. Thus, to explore these relationships in schizophrenia, we used the Scale for the Assessment of Negative Symptoms (SANS) and the Scale for Assessment of Positive Symptoms (SAPS), each of which provides a rather detailed assessment of specific clusters of negative and positive symptoms [32,33]. As such, these comprehensive rating scales provide yet another tool to test relationships among specific symptoms with personality and intelligence within the same sample of patients with schizophrenia, which in turn may offer critical data towards elucidating the nature of social cognition disturbance in schizophrenia.

A second aim of the current study focused on exploring the neural underpinnings of these individual differences in cognition, personality, and symptoms. To do so, we reanalyzed data from a subset of participants in the current investigation who had also undergone previous MRI studies of the fusiform gyrus (FG) and superior temporal gyrus (STG) [34]. Our question here is thus post hoc and asks how might variation in these neural structures that have been linked to social communication mapped onto personality and symptom expression in schizophrenia. That is, in our prior MRI studies in schizophrenia, we examined the relationship of symptoms with MRI gray matter volumes in these [34] and other related areas of the social brain, such as the orbital and prefrontal regions [35, 36], but we have yet to examine the intersection of symptoms, personality, and gray matter volumes within 
the same group of patients. Here we now present re-analyzed data that examines the relationship of individual variation in FG and STG gray matter volume with individual variation in symptom ratings as well as neuropsychological and personality tests within the same group of patients diagnosed with chronic schizophrenia.

\section{Material and Methods}

\section{Participants}

All research participants $(\mathrm{N}=111)$ were between the ages of 17 and 55 years, with a mean age of 39.53 ( $\mathrm{SD}=9.78)$, right-handed, native speakers of English, without histories of ECT, neurological illness, or alcohol or drug abuse in the past 5 years. All research participants gave informed consent prior to their participation in the study. Diagnoses for patients were ascertained by the Structured Clinical Interview for DSM-IV Axis I Disorders-Patient Edition (SCID-P), along with chart review. All patients were part of an ongoing comprehensive, longitudinal study of schizophrenia, and all were receiving neuroleptic medication; the mean chlorpromazine (CPZ) equivalent daily dose was $483.75 \mathrm{mg}(\mathrm{SD}=365.93)$. The mean duration of illness was 17.33 years $(\mathrm{SD}=10.96)$ and mean level of education was 13.53 years $(\mathrm{SD}=2.21)$. The sample consisted of 111 patients who completed the Wechsler Adult Intelligence-Scale-Third Edition (WAIS-III), 98 of these patients also completed the Revised NEO Personality Test (NEO-PI-R) and 89 also had available SANS and SAPS ratings.

\section{Measures}

The Wechsler Adult Intelligence-Scale-Third Edition (WAIS-III) yielded composite measures of intelligence (FullScale IQ, Verbal IQ, Performance IQ) and index scores of verbal comprehension, perceptual organization, working memory, and processing speed [37]. The Revised NEO Personality Test (NEO-PI-R) is an objective, self-report measure of five distinct and presumably universal personality traits: neuroticism, extraversion, openness, agreeableness, and conscientiousness [25]. DeYoung et al. [26] reported alpha reliabilities of internal consistency for the five NEO trait scales as .92 for Neuroticism; .87 for Extraversion; .89 for Openness; .91 for Agreeableness; and .91 for Conscientiousness. The Scale for the Assessment of Negative Symptoms (SANS) [32] and the Scale for Assessment of Positive Symptoms (SAPS) [33] are clinical assessment instruments that are widely used to rate negative and positive symptoms in schizophrenia. The SANS consists of 19 items representing five rationally-derived subscales: Affective Flattening or Blunting, Alogia, AvolitionApathy, Anhedonia-Asociality, and Inattention. The SAPS consists of 30 items representing four rationally-derived subscales: Hallucinations, Delusions, Bizarre Behavior, and Positive Formal Thought Disorder. Each subscale yields a score, a Likert rating of severity ranging from $0=$ none, $1=$ questionable, $2=$ mild, $3=$ moderate, $4=$ marked, and $5=$ severe. Empirical studies have shown that negative symptoms can be reliably distinguished from positive symptoms [38]. However, studies using factor analysis have pointed to a much more complex, multidimensional symptom structure, with three-or five-factor models marshalling the most empirical support $[38,39]$. Accordingly, we used the five SANS scores and the four SAPS scores to rate symptoms in schizophrenia.

\section{MRI processing}

MRI data were available for 22 right-handed, participants who served in prior studies of schizophrenia [40, 41]. The MRI protocol, described in detail in other published studies [40, 41], acquired MR images with a 1.5-Tesla General Electric scanner (GE Medical Systems, Milwaukee) at the Brigham and Women's Hospital in Boston (see Figure 3). Manual drawings of the FG were performed on the coronal plane, blind to diagnoses. The anterior landmark was reliably defined by one slice posterior to the mammillary body, and the posterior landmark was determined by the anterior tip of the parietal-occipital sulcus in the mid-sagittal plane. The last slice including the crux of the fornix provided the boundary for subdivision of FG into anterior and posterior. The collateral sulcus was used as the medial border. The occipital-temporal sulcus was used to determine the lateral border. Interrater reliability was computed for the FG by 3 independent raters who were blind to group membership. The intra-class correlations were: 0.94 for left anterior FG, 0.94 for the right anterior, 0.95 for the left posterior, 0.95 for the right posterior. For the STG, the anterior boundary was defined as the first slice containing the intact temporal stem. The posterior landmark was determined by the last slice including the crux of the fornix. Superior temporal sulcus was used as the inferior border. Inter-rater reliability was computed for the STG by 3 independent raters, blind to group membership. The intraclass correlations for inter-rater reliability were: 0.97 for left STG, 0.98 for right STG.

\section{Results}

We used the median WAIS-IV Full-Scale IQ score $($ median $=93.00)$ to divide the patients into low-IQ $(n=53)$ and high-IQ $(\mathrm{n}=58)$ groups. The two groups did not differ significantly in age: Low IQ (M = 40.84 years, $\mathrm{SD}=9.51)$, High IQ $(M=41.40$ years, $S D=10.51)$, duration of illness: Low IQ $(\mathrm{M}=16.74$ years, S.D. = 10.71), High IQ $(\mathrm{M}=17.84$ years, S.D. = 11.24), or chlorpromazine dosage equivalents: Low IQ $(\mathrm{M}=450.74 \mathrm{mg}, \mathrm{S} . \mathrm{D} .=291.66)$, High IQ $(\mathrm{M}=516.00 \mathrm{mg}$, S.D. = 427.33). However, the low-IQ group had significantly fewer years of education $(M=12.42$, S.D. $=1.88)$ than did the high-IQ group $(\mathrm{M}=14.52$, S.D. $=2.03), \mathrm{t}(108)=-5.60, \mathrm{p}<$ .001 . Table 1 presents personality test scores as well as positive and negative symptoms ratings for the two groups. As shown in Table 1, low-IQ and high-IQ groups showed similar levels of positive and negative symptoms. However, low-IQ and high-IQ groups showed different NEO personality patterns as reflected in the highly statistically significant group $\mathrm{x}$ personality traits interaction, $\mathrm{F}(4,328)=5.09, \mathrm{p}=.001$, Partial Eta Squared $=.058$. In comparison to the high-IQ group, the low-IQ group had elevated neuroticism, $\mathrm{t}(82)=-3.02, \mathrm{p}=.013$ and reduced levels of openness, $\mathrm{t}(82)=-3.02, \mathrm{p}=.003$ (see Figure 1). Personality-symptoms correlations indicated that 
among the five NEO personality traits, only formal thought disturbance correlated with NEO personality traits of lower neuroticism $(\mathrm{r}=-.223, \mathrm{p}=.037)$ and higher openness $(\mathrm{r}=.242$, $\mathrm{p}=.023)$.

Table 1: NEO personality test scores and SAPS/SANS ratings for lowIQ and high-IQ patients with schizophrenia.

\begin{tabular}{|l|l|l|}
\hline \multicolumn{3}{|c|}{ Schizophrenia } \\
\hline Measures & Low IQ & High IQ \\
\hline NEO-PI-R & $23.08( \pm 9.57)$ & $16.64( \pm 10.43)^{*}$ \\
\hline Neuroticism & $25.25( \pm 6.68)$ & $28.18( \pm 8.18)$ \\
\hline Extraversion & $24.23( \pm 5.25)$ & $27.82( \pm 6.57)^{* *}$ \\
\hline Openness & $31.44( \pm 6.35)$ & $33.74( \pm 6.34)$ \\
\hline Agreeableness & $30.19( \pm 7.21)$ & $33.96( \pm 7.20)$ \\
\hline Conscientiousness & & \\
\hline SAPS & $2.8095( \pm 1.91)$ & $2.398( \pm 1.74)$ \\
\hline Hallucinations & $3.0952( \pm 1.66)$ & $3.4286( \pm 1.35)$ \\
\hline Delusions & $1.5000( \pm 1.36)$ & $1.1633( \pm 1.29)$ \\
\hline Bizarre Behavior & $1.6667( \pm 1.58)$ & $1.7959( \pm 1.63)$ \\
\hline Formal Thought Disorder & & \\
\hline SANS & $2.2889( \pm 1.72)$ & $2.3068( \pm 1.55)$ \\
\hline Affective Flattening & $1.9333( \pm 1.51)$ & $1.9091( \pm 1.59)$ \\
\hline Alogia & $2.6667( \pm 1.59)$ & $2.7273( \pm 1.46)$ \\
\hline Avolition-Apathy & $2.9111( \pm 1.56)$ & $3.1818( \pm 1.28)$ \\
\hline Anhedonia-Asociality & $2.1444( \pm 1.67)$ & $2.0682( \pm 1.63)$ \\
\hline Attention & &
\end{tabular}

${ }^{*} \mathrm{p}<.05,{ }^{* *} \mathrm{p}<.01$

Note: Values are means plus or minus standard deviations

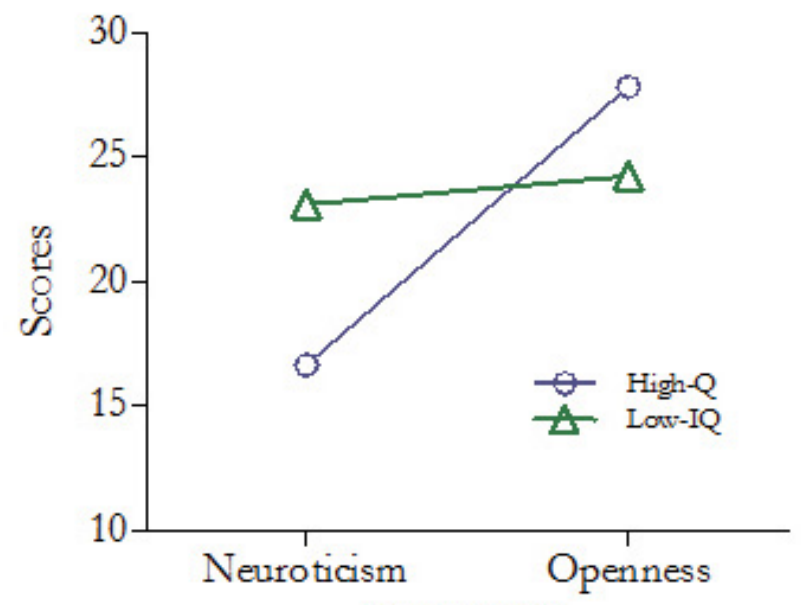

NEO-PI-R

Figure 1: Neuroticism and openness scores for high-and low IQ patients with schizophrenia.

We next tested the interaction of these differences in personality traits with symptoms in high- and low-IQ groups. In a mixed-model ANOVA with one between-subjects factor of group (low-IQ, high-IQ) and two within-subjects factors of personality (neuroticism, openness) and symptoms (positive, negative), the three-way interaction of intelligence $\mathrm{x}$ personality $\mathrm{x}$ symptoms provided a direct statistic test of our hypothesis that high-IQ and low-IQ groups would show different patterns of both symptoms and personality traits. Specifically, a mixed-model ANOVA revealed a highly statistically significant three-way interaction of group (high-IQ, low-IQ) x personality (Neuroticism, Openness) $\mathrm{x}$ symptoms (positive, negative), $\mathrm{F}(1,77)=11.95, \mathrm{p}=.001$, Partial Eta Squared = .134. Planned follow-up comparisons suggested that this three-way interaction was driven primarily by personality differences especially in markedly reduced openness, $\mathrm{t}(77)=-3.68, \mathrm{p}<.001$ and to a lesser extent but still statistically significant higher level of neuroticism, $\mathrm{t}(77)=$ 2.12, $\mathrm{p}=.037$ for the low-IQ group.

In addition, ANOVA revealed a highly significant twoway interaction of personality x symptoms, $F(1,77)=30.29 p$ $<.001$, Partial Eta Squared = .282, due largely to the differential effects the positive symptom of formal thought disturbance had on personality traits of neuroticism and openness. For example, a mixed-model ANOVA with one between-subject factor of thought disturbance (low-thought disturbance, high-thought disturbance, based on median split) and one within-subjects factor of personality traits (neuroticism, openness) revealed a statistically significant interaction of thought disturbance $\mathrm{x}$ personality traits, $\mathrm{F}(1,86)=7.27, \mathrm{p}$ $=.008$, Partial Eta Squared $=.101$. As shown in Figure 2, in comparison to the high-thought disturbance group, the lowthought disturbance group had higher neuroticism and lower openness.

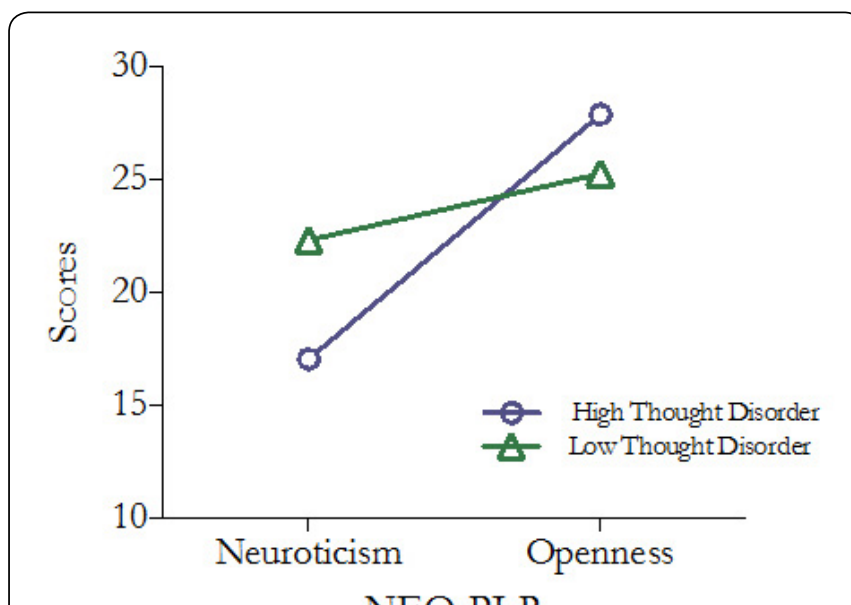

Figure 2: Neuroticism and openness scores of high and low thought disordered patients with schizophrenia.

\section{MRI correlations}

We next examined MRI gray matter correlation coefficients with personality and symptoms that met statistical significance at a level adjusted using Bonferroni correction for multiple comparisons [42]. Left posterior STG gray matter volume correlated with both the personality trait of agreeableness $(\mathrm{r}=.658, \mathrm{p}=.002)$ and the positive symptom ratings of hallucinations $(\mathrm{r}=-720, \mathrm{p}<.001)$. These correlations indicated that smaller left posterior STG gray matter volume was associated with both lower agreeableness and more severe 
hallucinations. By comparison, left posterior FG gray mater correlated with both openness $(\mathrm{r}=.725, \mathrm{p}<.001)$ and negative symptom ratings of social anhedonia $(r=-.568, \mathrm{p}=.006)$. These correlations indicated that reduced left posterior FG was associated with both lower openness and more severe social anhedonia. Figure 3 presents scatter plots of these significant correlations of brain regions with personality traits and symptom ratings.
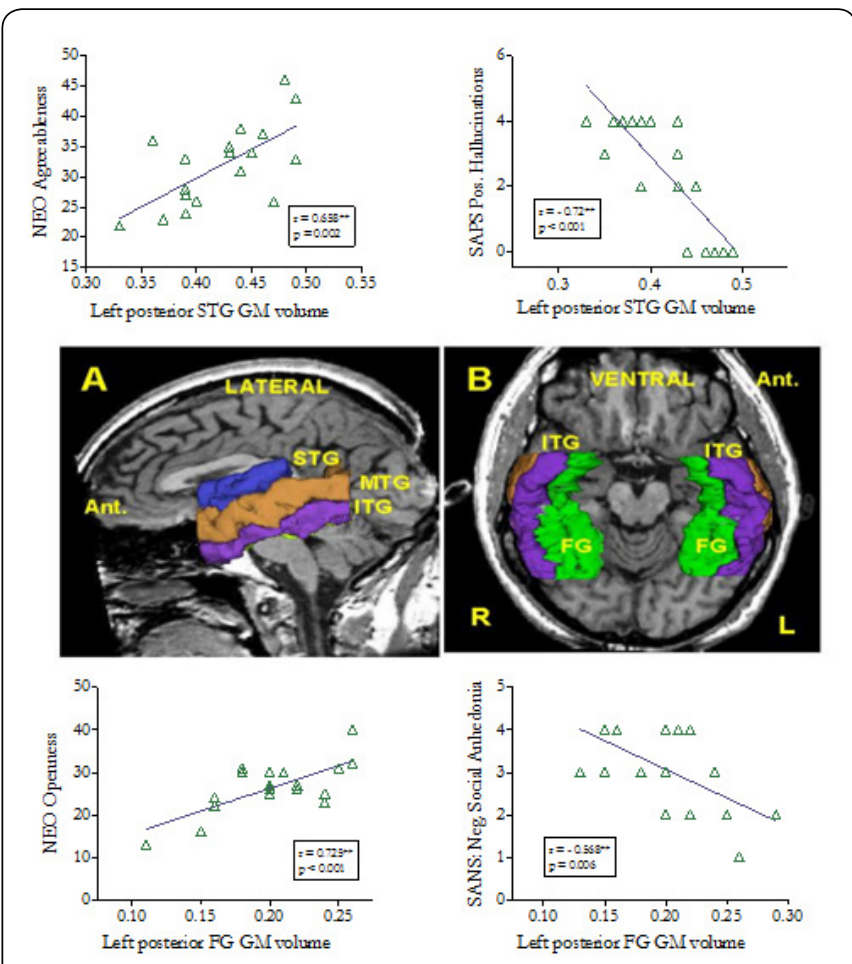

Figure 3: Scatter plots of left posterior superior temporal gyrus (LPSTG) and left posterior fusiform gyrus (LPFG) with personality traits and symptom ratings.

Finally, we used hierarchical regression to examine the unique and joint contributions of personality traits and symptoms to MRI gray matter volumes. Hierarchical regression results indicated that agreeableness $(B=.361, \mathrm{p}=$ $.048)$ and positive symptoms of hallucinations $(B=-.600, \mathrm{p}=$ .003 ) each accounted for a significant portion of variance in left posterior STG volume. Agreeableness accounted for 9.92\% to $25.10 \%$ of the variance in left posterior STG gray matter volume, as reflected by a semi-partial correlation value of .315 and a partial correlation value of .501 , and positive symptoms related to hallucinations accounted for an additional $27.46 \%$ to $48.02 \%$ of the variance in left posterior STG gray matter volume, as reflected by a semi-partial correlation value of -.524 and a partial correlation value of -.693. For left posterior FG, joint contributions of openness and social anhedonia reached statistical significance, $\mathrm{F}(2,17)=6.01, \mathrm{p}=.012$, but each alone did not.

\section{Discussion}

The current study combined neuropsychological and personality measures, along with symptoms ratings in order to examine the nature of social cognitive disturbance in schizophrenia. In addition, a subset of patients had available prior structural brain MRI studies of the FG and STG, two key regions that play important roles in social cognition [13]. Behaviorally, the results provided strong statistical evidence of the interacting influences of intelligence, personality and symptoms in social cognitive disturbance in schizophrenia. The MRI results, although limited by the small size of the sample subset, extended these behavioral findings by demonstrating gray matter volumes for the STG and FG were each associated with specific personality-symptoms relationships. These behavioral and MRI findings are discussed below.

First, with respect to the behavioral data, the current findings offered evidence of a particularly strong relationship between intelligence and personality in this sample of patients with chronic schizophrenia. While intelligence has long been known to be deleteriously affected by schizophrenia, its relationship to other important facets of the illness has not been as well studied. In this regard, the current study revealed a highly statistically significant interaction of intelligence, personality, and symptoms. This interaction was largely if not exclusively attributed to different patterns of personality traits for high- and low-IQ groups of patients with schizophrenia. The differences were most pronounced for personality traits of neuroticism and openness where low-IQ patients showed elevated neuroticism and reduced openness in comparison with high-IQ patients.

Each of these personality traits may be linked to the clinical phenotype of schizophrenia, but in different ways. For example, Jones et al. [43] recently noted that genetic risk for schizophrenia in adolescence is often expressed as anxiety and negative symptoms. These researchers called for a greater focus on these emotional processes rather than psychotic experiences as candidate disease endophenotypes. In a similar vein, Holt et al. [44] suggested that emotional activation and regulation may play a critical role in the development of schizophrenia. In line with this reasoning, the current findings of heightened neuroticism and lower intelligence may be understood as reflecting the emotional burden of full-blown, clinically diagnosable schizophrenia, perhaps indicative of the endpoint of a disease-related developmental trajectory first identified by these risk studies. This emotional burden, suggested by the current findings, may be expressed by a combination of intellectual decline, anxiety-related personality facets of neuroticism, as well as motivation-based deficits of negative symptoms.

On the other hand, the strong link of intelligence and openness, observed by the current findings, may capture a distinct dimension of the illness that is very different from, yet equally as important as that of anxiety-related neuroticism and negative symptoms. Consider, for example, that the current data revealed statistically significant relationships between positive symptoms of formal thought disorder and personality traits marked by high openness and low neuroticism. Consider also that in healthy samples, openness has been linked to both intelligence and creativity [45, 46]. Consider, too, clinical studies of personality disorders that have associated high 
openness with eccentric thinking, fantasy, and daydreaming as well as with tenuous reality testing, susceptibility to altered states of consciousness and hallucinatory experiences, in contrast to low openness that has been associated with emotional blandness, cognitive inflexibility, and limited initiative and motivation [47].

To our knowledge, the current findings represent one of the first studies to link individual differences in personality and symptoms in schizophrenia, specifically increased severity of thought disturbance with high openness and low neuroticism. In so doing, these findings may also have implications for studies that have long sought to understand the relationship of psychiatric disorders with increased creativity [48, 49]. To this end, our data suggest that the personality trait of openness, a well-established correlate of creativity, is accompanied by heightened thought disorder which was measured by the SAPS and is defined, in part, by a fluidity of loosely connected ideas. This leads, in turn, to the intriguing possibility that creativity and psychiatric disorders, in general, and thought disturbance in schizophrenia, in particular, might be associated through a common variation in the personality trait of openness.

With respect to the MRI data, results point to dissociable personality-symptoms relationships for FG versus STG in schizophrenia. As such, these data represent one of the first to show how variation in both personality and symptoms may be differentially related to variation in structural anatomy of the FG and STG. That is, left posterior gray mater volumes of the left posterior FG, but not the left posterior STG, were associated with both heightened SANS ratings of social anhedonia and low scores on NEO trait of openness, whereas left posterior STG but not left posterior FG left posterior gray matter volumes, correlated with both increased SAPS ratings of hallucinations and low score for the NEO personality trait of agreeableness. Unlike the larger sample, these relationships were not influenced by intelligence in this smaller subset of patients.

These MRI data thus suggest that FG and STG may each make distinct contributions to disease-related changes in social cognition. For example, the FG has long been associated with face perception, considered a basic building block of social cognition that is important for reading emotions and social signals in the environment $[18,50]$. In addition, fMRI studies have linked social anhedonia with neural abnormalities in face emotion processing regions of the brain in both healthy participants living in the community [51] and in patients with schizophrenia [52]. The FG contribution may be seen primarily in terms of disease-related deficits in social motivation --- desire and pleasure of social connection - as well as in intellectual curiosity. In the current study, these FG-linked deficits were reflected, respectively, by heightened negative symptoms related to social anhedonia and low NEO scores on the personality trait of openness to experience.

By comparison, studies of patients with schizophrenia have linked STG to various positive symptoms, including auditory hallucinations, paranoid delusions, and formal thought disorder [34, 53-56]. The current data extend these findings by linking left posterior STG volume to not only heightened hallucinations but also to the personality trait of agreeableness as measured by the NEO. Of the five NEO personality traits, agreeableness is considered to be the most salient index of human sociality, and it is presumed to capture underlying psychological mechanisms that facilitate the understanding and appreciation of others' emotions, mental states, and motivations related to empathy, theory of mind, and other expressions of social information processing $[26,57,58]$. In this regard, then, the current findings extend those of prior studies linking STG with positive symptoms and raise the intriguing question that positive symptoms and personality processes related to agreeableness may share common neural sources in STG circuitry. Indeed, our results show that agreeableness and positive symptoms account for an exceptionally large portion of the variance, ranging from $44.61 \%$ to $66.71 \%$, in left posterior STG gray matter volume.

In summary, there are several limitations in this current study that aimed to examine the social brain hypothesis using a comprehensive battery of measures of intelligence, personality, and symptoms in a sample of medicated patients with long term, chronic schizophrenia. First, while serving as highly and reliable indexes of key characteristics of the illness, these standardized assessment tools provide proxy rather than direct measures of social cognition. In this regard, experimental probes of particular perceptual and affective operations would have complemented these psychometric measures, thereby offering a more precise understanding of social cognitive processes in schizophrenia. In a similar vein, the MRI data came from a very small subset of participants who had available prior studies of the STG and the FG, and thus these data are viewed as offering only post hoc analyses of variations in these neural structures with personality and symptom expression in schizophrenia. Likewise, while the results revealed several significant findings, the generalizability of these data is limited to persons with chronic schizophrenia with long histories of neuroleptic treatment similar to the research participants in this study. In addition, the study focused on social cognition within schizophrenia and divided the sample into high- and low-IQ groups as a means to address the well-known problem of heterogeneity of illness expression. However, the study did not include a control group of healthy participants that would allow for comparisons with the patient sample. This too represented a limitation in the research design of the study. And, finally an additional limitation of this study relates to the difficulty in attributing the observed findings to the consequences of the illness per se versus confounding factors such as those related to treatment, nutrition, health, etc. Nonetheless, the current findings offer a novel approach to the study of social cognition in schizophrenia. Moreover, the observed findings may help to define a testable model of social cognition that can be applied in prospective studies ranging the spectrum of at-risk, prodromal, and first episode subjects.

\section{References}

1. Heinrichs RW. 2005. The primacy of cognition in schizophrenia. Am Psychol 60(3): 229-242. https://doi.org/10.1037/0003-066X.60.3.229

2. Kraepelin E. 1971. Dementia Praecox and Paraphrenia. Huntington, New York, USA. 
3. Bleuler E. 1924. Textbook of psychiatry. Macmillan, New York, USA.

4. Kretschmer AA. 1934. A text-book of medical psychology. Oxford University Press, Oxford, UK.

5. Meehl PE. 1962. Schizotaxia, schizotypy, schizophrenia. Am Psychol 17(12): 827-838. https://doi.org/10.1037/h0041029

6. Meehl PE. 1975. Hedonic capacity: some conjectures. Bull Menninger Clin 39(4): 295-307.

7. Meehl PE. 1989. Schizotaxia revisited. Arch Gen Psychiatry 46(10): 935-944. https://doi.org/10.1001/archpsyc.1989.01810100077015

8. Brunet-Gouet E, Decety J. 2006. Social brain dysfunctions in schizophrenia: a review of neuroimaging studies. Psychiatry Res 148(23): 75-92. https://doi.org/10.1016/j.pscychresns.2006.05.001

9. Burns JK. 2004. An evolutionary theory of schizophrenia: cortical connectivity, metarepresentation, and the social brain. Behav Brain Sci 27(6): 831-55. https://doi.org/10.1017/S0140525X04000196

10. Burns J K. 2006. Psychosis: a costly by-product of social brain evolution in Homo sapiens. Prog Neuropsychopharmacol Biol Psychiatry 30(5): 797814. https://doi.org/10.1016/j.pnpbp.2006.01.006

11. Crespi B, Badcock C. 2008. Psychosis and autism as diametrical disorders of the social brain. Behav Brain Sci 31(3): 241-261. https:// doi.org/10.1017/S0140525X08004214

12. Green MF, Horan WP, Lee J. 2015. Social cognition in schizophrenia. Nat Rev Neurosci 16(10): 620-631. https://doi.org/10.1038/nrn4005

13. Nestor PG, Choate V, Shirai A. 2015. In search of the functional neuroanatomy of social disturbance in schizophrenia. In: Schutt RK, Seidman LJ, Keshavan MS (eds) Social neuroscience: brain, mind, and society. Harvard University Press, Cambridge, MA, USA, pp 88-122.

14. Duchaine B Cosmides L, Tooby J. 2001. Evolutionary psychology and the brain. Curr Opin Neurobiol 11(2): 225-230. https://doi.org/10.1016/ S0959-4388(00)00201-4

15. Adolphs R.2003. Cognitive neuroscience of human social behaviour. Nat Rev Neurosci 4(3): 165-178. https://doi.org/10.1038/nrn1056

16. Amodio DM, Frith, CD. 2006. Meeting of minds: the medial frontal cortex and social cognition. Nat Rev Neurosci 7(4): 268-277. https://doi. org/10.1038/nrn1884

17. Nestor PG, Nakamura M, Niznikiewicz M, Thompson E, Levitt JJ, et al. 2013. In search of the functional neuroanatomy of sociality: MRI subdivisions of orbital frontal cortex and social cognition. Soc Cogni Affect Neurosci 8(4): 460-467. https://doi.org/10.1093/scan/nss018

18. Brothers L. 1990. The neural basis of primate social communication. Motiv Emot 14(2): 81-91. https://doi.org/10.1007/bf00991637

19. Sallet J, Mars RB, Noonan MP, Andersson JL, O'Reilly JX, et al. 2011. Social network size affects neural circuits in macaques. Science 334(6056): 697-700. https://doi.org/10.1126/science.1210027

20. Herrmann E, Call J, Hernandez-Lloreda MV, Hare B, Tomasello M. 2007. Humans have evolved specialized skills of social cognition: the cultural intelligence hypothesis. Science 317(5843): 1360-1366. https:// doi.org/10.1126/science. 1146282

21. Herrmann E, Hare B, Call J, Tomasello M. 2010. Differences in the cognitive skills of bonobos and chimpanzees. PloS one 5(8): e12438. https://doi.org/10.1371/journal.pone.0012438

22. van Schaik CP, Isler K, Burkart JM. 2012. Explaining brain size variation: from social to cultural brain. Trends Cogn Sci 16(5): 277-284. https://doi.org/10.1016/j.tics.2012.04.004

23. Potter A, Nestor PG. 2010. IQ subtypes in schizophrenia: distinct symptom and neuropsychological profiles. J Nerv Ment Dis 198(8): 580-585. https://doi.org/10.1097/NMD.0b013e3181ea4e4

24. Fusar-Poli P, Bonoldi I, Yung AR, Borgwardt S, Kempton MJ, et al. 2012. Predicting psychosis: meta-analysis of transition outcomes in individuals at high clinical risk. Arch Gen Psychiatry 69(3): 220-229. https://doi.org/10.1001/archgenpsychiatry.2011.1472
25. Costa PT Jr., McCrae RR. 1992. Four ways five factors are basic. Pers Individ Dif 13(6): 653-665. https://doi.org/10.1016/01918869(92)90236-I

26. DeYoung CG, Hirsh JB, Shane MS, Papademetris X, Rajeevan N, et al. 2010. Testing predictions from personality neuroscience. Brain structure and the big five. Psychol Sci 21(6): 820-828. https://doi. org/10.1177/0956797610370159

27. Horan WP, Brown SA, Blanchard JJ. 2006. Social anhedonia and schizotypy: the contribution of individual differences in affective traits, stress, and coping. Psychiatry Res 149(1-3): 147-156. https://doi. org/10.1016/j.psychres.2006.06.002

28. Barrantes-Vidal N, Ros-Morente A, Kwapil TR. 2009. An examination of neuroticism as a moderating factor in the association of positive and negative schizotypy with psychopathology in a nonclinical sample. Schizophr Res 115(2-3): 303-309. https://doi.org/10.1016/j. schres.2009.09.021

29. Hoffman RE. 2007. A social deafferentation hypothesis for induction of active schizophrenia. Schizophr Bull 33(5): 1066-1070. https://doi. org/10.1093/schbul/sbm079

30. Valmaggia LR, Stahl D, Yung AR, Nelson B, Fusar-Poli P, et al. 2013. Negative psychotic symptoms and impaired role functioning predict transition outcomes in the at-risk mental state: a latent class cluster analysis study. Psychol Med 43(11): 2311-2325. https://doi.org/10.1017/ S0033291713000251

31. Masillo A,Day F,Laing J,Howes O, Fusar-Poli, et al.2012. Interpersonal sensitivity in the at-risk mental state for psychosis. Psychol Med 42(9): 1835-1845. https://doi.org/10.1017/S0033291711002996

32. Andreasen NC. 1983. Scale for the assessment of negative symptoms: SANS. Department of Psychiatry, College of Medicine, the University of Iowa, USA.

33. Andreasen NC. 1984. Scale for the assessment of positive symptoms: SAPS. Department of Psychiatry, College of Medicine, the University of Iowa, USA.

34. Nestor PG, Onitsuka T, Gurrera RJ, Niznikiewicz M, Frumin M, et al. 2007. Dissociable contributions of MRI volume reductions of superior temporal and fusiform gyri to symptoms and neuropsychology in schizophrenia. Schizophr Res 91(1-3): 103-106. https://doi. org/10.1016/j.schres.2006.11.025

35. Nakamura M, Nestor PG, Levitt JJ, Cohen A, Kawashima T, et al. 2008. Orbitofrontal volume deficit in schizophrenia and thought disorder. Brain 131(1): 180-195. https://doi.org/10.1093/brain/awm265

36. Ohtani T, Levitt JJ, Nestor PG, Kawashima T, Asami T, et al. 2014. Prefrontal cortex volume deficit in schizophrenia: a new look using 3T MRI with manual parcellation. Schizophr Res 152(1): 184-190. https:// doi.org/10.1016/j.schres.2013.10.026

37. Wechsler D. 2014. Wechsler Adult Intelligence Scale-Fourth Edition (WAIS-IV).

38. Blanchard JJ, Cohen AS. 2005. The structure of negative symptoms within schizophrenia: implications for assessment. Schizophr Bull 3(2): 238-245. https://doi.org/10.1093/schbul/sbj013

39. Grube BS, Bilder RM, Goldman RS. 1998. Meta-analysis of symptom factors in schizophrenia. Schizophr Res 31(2-3): 113-120. https://doi. org/10.1016/S0920-9964(98)00011-5

40. Onitsuka T, Shenton ME, Kasai K, Nestor PG, Toner SK, et al. 2003. Fusiform gyrus volume reduction and facial recognition in chronic schizophrenia. Arch Gen Psychiatry 60(4): 349-355. https://doi. org/10.1001/archpsyc.60.4.349

41. Onitsuka T, Shenton ME, Salisbury DF, Dickey CC, Kasai K, et al. 2004. Middle and inferior temporal gyrus gray matter volume abnormalities in schizophrenia: an MRI study. Am J Psychiatry 161(12): 1603-1611. https://doi.org/10.1176/appi.ajp.163.12.2103

42. Curtin F, Schulz P. 1998. Multiple correlations and Bonferroni's correction. Biol Psychiatry 44(8): 775-777. https://doi.org/10.1016/ S0006-3223(98)00043-2 
43. Jones HJ, Stergiakouli E, Tansey KE, Hubbard L, Heron J, et al. 2016. Phenotypic manifestation of genetic risk for schizophrenia during adolescence in the general population. JAMA Psychiatry 73(3): 221-228. https://doi.org/10.1001/jamapsychiatry.2015.3058

44. Holt DJ. 2016. A pathway to understanding emotional dysfunction in schizophrenia. JAMA Psychiatry 73(6): 555-556. https://doi. org/10.1001/jamapsychiatry.2016.0172

45. Harris JA. 2004. Measured intelligence, achievement, openness to experience, and creativity. Pers Individ Dif 36(4): 913-929. https://doi. org/10.1016/s0191-8869(03)00161-2

46. McCrae R. 1987. Creativity, divergent thinking, and openness to experience. J Pers Soc Psychol 52(6): 1258-1265. https://doi. org/10.1037/0022-3514.52.6.1258

47. Costa PT, Widiger TA. 2002. Personality disorders and the five-factor model of personality. American Psychological Association, Washington, DC, USA.

48. Keller MC, Visscher PM. 2015. Genetic variation links creativity to psychiatric disorders. Nat Neurosci 18(7): 928-929. https://doi. org/10.1038/nn.4047

49. Power RA, Steinberg S, Bjornsdottir G, Rietveld CA, Abdellaoui A, et al. 2015. Polygenic risk scores for schizophrenia and bipolar disorder predict creativity. Nat Neurosci 18(7): 953-955. https://doi.org/10.1038/ nn.4040

50. Pinkham AE, Penn DL, Perkins DO, Lieberman J. 2003. Implications for the neural basis of social cognition for the study of schizophrenia. Am J Psychiatry 160(5): 815-824. https://doi.org/10.1176/appi. ajp.160.5.815

51. Germine LT, Garrido L, Bruce L, Hooker C. 2011. Social anhedonia is associated with neural abnormalities during face emotion processing. Neuroimage 58(3): 935-945. https://doi.org/10.1016/j. neuroimage.2011.06.059

52. Lepage M, Sergerie K, Benoit A, Czechowska Y, Dickie E, et al. 2011. Emotional face processing and flat affect in schizophrenia: functional and structural neural correlates. Psychol Med 41(9): 1833-1844. https:// doi.org/10.1017/S0033291711000031

53. Barta PE, Pearlson GD, Powers RE, Richards SS, Tune LE. 1990. Auditory hallucinations and smaller superior temporal gyral volume in schizophrenia. Am J Psychiatry 147(11): 1457-1462. https://doi. org/10.1176/ajp.147.11.1457

54. Marsh L, Harris D, Lim KO, Beal M, Hoff AL, et al. 1997. Structural magnetic resonance imaging abnormalities in men with severe chronic schizophrenia and an early age at clinical onset. Arch Gen Psychiatry 54(12): 1104-1112. https://doi.org/10.1001/ archpsyc.1997.01830240060009

55. McCarley RW, Shenton ME, O’Donnell BF, Faux SF, Kikinis R, et al. 1993. Auditory P300 abnormalities and left posterior superior temporal gyrus volume reduction in schizophrenia. Arch Gen Psychiatry 50(3): 190-197. https://doi.org/10.1001/archpsyc.1993.01820150036003

56. Shenton ME, Kikinis R, Jolesz FA, Pollak SD, LeMay M, et al. 1992. Abnormalities of the left temporal lobe and thought disorder in schizophrenia. A quantitative magnetic resonance imaging study. $N$ Engl J Med 327(9): 604-612. https://doi.org/10.1056/ NEJM199208273270905

57. Graziano WG, Habashi MM, Sheese BE, Tobin RM. 2007. Agreeableness, empathy, and helping: a person x situation perspective. $J$ Pers Soc Psychol 93(4): 583-599. https://doi.org/10.1037/00223514.93.4.583

58. Nettle D, Liddle B. 2008. Agreeableness is related to social-cognitive, but not social-perceptual, theory of mind. Eur J Pers 22(4): 323-335. https://doi.org/10.1002/per.672 